\title{
Dependence and Addiction During Chronic Opioid Therapy
}

\author{
David N. Juurlink • Irfan A. Dhalla
}

Published online: 17 October 2012

(C) American College of Medical Toxicology 2012

\begin{abstract}
The use of opioids for chronic noncancer pain has increased dramatically over the past 25 years in North America and has been accompanied by a major increase in opioid addiction and overdose deaths. The increase in opioid prescribing is multifactorial and partly reflects concerns about the effectiveness and safety of alternative medications, particularly the nonsteroidal anti-inflammatory drugs. However, much of the rise in opioid prescribing reflects the assertion, widely communicated to physicians in the 1990s, that the risks of dependence and addiction during chronic opioid therapy were low, predictable, and could be minimized by the use of controlled-release opioid formulations.
\end{abstract}

D. N. Juurlink $(\bowtie)$

Sunnybrook Research Institute,

Toronto, Canada

e-mail: david.juurlink@ices.on.ca

I. A. Dhalla

Li Ka Shing Knowledge Institute of St. Michael's Hospital, Toronto, Canada

D. N. Juurlink $\cdot$ I. A. Dhalla

Department of Medicine, University of Toronto,

Toronto, Canada

D. N. Juurlink

Department of Pediatrics, University of Toronto,

Toronto, Canada

D. N. Juurlink $\cdot$ I. A. Dhalla

Institute of Health Policy, Management, and Evaluation,

University of Toronto,

Toronto, Canada

D. N. Juurlink $\cdot$ I. A. Dhalla

Institute for Clinical Evaluative Sciences,

Toronto, Canada

D. N. Juurlink

Sunnybrook Health Sciences Centre,

G Wing 106, 2075 Bayview Avenue,

Toronto, ON M4N 3M5, Canada
In this narrative review, we offer a critical appraisal of the publications most frequently cited as evidence that the risk of addiction during chronic opioid therapy is low. We conclude that very few well-designed studies support the notion that opioid addiction is rare during chronic opioid therapy and that none can be readily generalized to present-day practice. Despite serious methodological limitations, these studies have been repeatedly mischaracterized as showing that the risk of addiction during chronic opioid therapy is rare. These studies are countered by a larger, more rigorous and contemporary body of evidence demonstrating that dependence and addiction are relatively common consequences of chronic opioid therapy, occurring in up to onethird of patients in some series.

Keywords Opioids $\cdot$ Addiction $\cdot$ Dependence $\cdot$ Review

\section{Introduction}

Chronic pain is a problem for millions of patients, many of whom have disabling symptoms that interfere with day-to-day function at home and in the workplace [1]. While various nonpharmacologic options (such as cognitive-behavioral therapy, supervised exercise and massage) are helpful for some patients with chronic pain [2] and while surgical treatment (such as joint replacement, regional nerve blocks or spinal cord stimulation) is an option for others, many of these interventions are more difficult to obtain, more costly and are inconsistently covered by private and public health insurance. As a result, medications are used early in the management of chronic pain for many patients. Unfortunately, the available drug options are limited and consisting primarily of acetaminophen, nonsteroidal anti-inflammatory drugs (NSAIDs) and opioids. Other medications, including carbamazepine, gabapentin and antidepressants afford benefit for some types of pain. 
The use of opioids for pain due to acute conditions (e.g., fractures, renal colic and postoperative pain) and at the end of life (e.g., metastatic cancer) has been widely accepted for decades, but the same is not true of chronic noncancer pain [3]. In North America, it was not until the late 1980s and, in particular, the 1990s that opioids became a commonplace element of therapy for patients with chronic noncancer pain [4]. The rise in opioid use coincided with the introduction of several new opioid formulations, most notably OxyContin, a controlled release formulation of oxycodone introduced in the USA in 1995 and in Canada in 1996.

The move toward opioids was gradual and reflected a confluence of several factors, including observations regarding the safety and effectiveness of opioids for more widely accepted indications [5], reservations about the long-term safety of alternate therapies (particularly NSAIDs in the elderly), the aggressive promotion of opioids (and OxyContin in particular) to frontline clinicians, and endorsements by national organizations including the American Academy of Pain Medicine (AAPM) and the American Pain Society (APS). Indeed, in 1996, these organizations adopted a consensus statement encouraging the more liberal use of opioids for chronic pain, stating that "studies indicate that the de novo development of addiction when opioids are used for the relief of pain is low" [6]. As noted by Ballantyne [7], the potential benefits of opioids coupled with the assertion of minimal risk created an ethical imperative to use these drugs more freely for chronic noncancer pain. Indeed, even the Joint Commission (formerly the Joint Commission on Accreditation of Healthcare Organizations) recommended that in hospitalized patients, pain be regularly evaluated as the "fifth vital sign" [8], even though pain is a symptom rather than a sign, and cannot be measured as objectively as true vital signs such as heart rate and temperature.

\section{Historical Perspective}

Opium, the complex mixture of alkaloids and other chemicals derived from Papaver somniferum, has been used for medicinal purposes since prehistoric times. The addictive potential of opium has also long been recognized. For example, more than 2,000 years ago Diagoras concluded that it was better to suffer pain than to develop an addiction to opium [9]. Hippocrates, in contrast, believed that opium should be prescribed to some patients but only with great caution.

Opium was widely available in the eighteenth and nineteenth centuries and used for a variety of reasons, including diarrhea, pain and insomnia. Although some Chinese authorities recognized the potential for widespread addiction in the early eighteenth century, their concerns did not initially result in import restrictions. A century later, with the majority of the adult population in some coastal Chinese provinces dependent on opium, government officials became concerned not only about public health but also about the impact of opium use on the economy. Their attempts to limit the opium trade eventually led to the Opium Wars (1839-1842 and 1856-1860), both of which were won by Britain.

Despite its use as a poison, the use of opium did not become a major public health concern in the USA until the latter half of the nineteenth century. In 1915, Charles Towns claimed that approximately $15 \%$ of American physicians were addicted to the drug [10]. While this figure seems improbably high, concerns about addiction and its negative effects were pervasive in the early 1900 s, leading to legislation in many countries along with profound changes in clinical practice. The reticence of physicians to prescribe opioids was so strong that many patients with pain due to terminal cancer suffered needlessly. Indeed, in some countries, and sometimes even in North America, patients with pain from metastatic cancer continue to receive insufficient analgesia due to a misplaced fear of addiction. In this context, the desire of patients and physicians to increase accessibility to opioids is easily understood. Unfortunately, societal amnesia has led to the rediscovery of the potential for harm when opioids are used for long periods of time

\section{Terminology}

Fishbain and colleagues have noted that at least some of the difficulty in characterizing the unintended consequences of chronic opioid therapy relates to inconsistency, overlap and disagreement regarding commonly used terms such as addiction [11]. The lack of uniform terminology along with misunderstanding and misapplication of commonly used terms frequently creates problems when comparing and interpreting literature on the issue of opioid addiction. Some definitions have evolved over time, some exist on a spectrum rather than conditions that are simply present or not, and some remain controversial. Indeed, it is anticipated that the fifth edition of the Diagnostic and Statistical Manual of Mental Disorders (DSM-5) will eliminate disease categories for substance abuse and dependence, replacing them with "addictions and related disorders" in part because of overlap and confusion with the associated terms. With these caveats in place, a brief overview of some terms in widespread use is warranted. A more detailed exposition of this is provided by Hernandez and Nelson [12].

Nonmedical Use The term nonmedical use refers to the situation in which an individual uses a prescription drug without an actual prescription, or for the psychological feelings produced by the drug [13]. A common example of 
nonmedical opioid use would be a teen who takes a prescription opioid provided by a friend to obtain the pleasurable effects of the drug.

Misuse Denotes situations in which a patient uses a prescribed medication, deliberately or unintentionally, in a manner or for a reason different from that for which it was prescribed. For example, a patient may choose to take a controlled-release opioid on an as-needed basis, rather than at regularly scheduled intervals. Alternatively, a patient may take an opioid at bedtime to facilitate sleep.

Drug Abuse The term drug abuse implies a maladaptive pattern of drug use that is not condoned or supervised by a physician, either with regard to the amount used, the method of use, or both. Abuse involves elements of physical, psychological, financial, legal or social harm, either to the user or others affected by the user's behavior, including family members or coworkers. For example, an individual may surreptitiously obtain opioids from multiple physicians, or crush the tablets to defeat a controlled-release mechanism to increase the sense of euphoria.

Drug Addiction Is a chronic and frequently relapsing disorder characterized by compulsive drug-seeking and persistent use despite ongoing harm of a physical, psychological or social nature. The American Society for Addiction Medicine also notes that opioid addiction is characterized by a loss of control over drug use and a preoccupation with obtaining opioids despite adequate analgesia [14]. Importantly, the term addiction incorporates the consequences of use, including tolerance and withdrawal, persistent unsuccessful attempts to reduce use, and reduction or elimination of important social, occupational, or recreational activities as a result of drug use. For example, an individual prescribed an opioid for chronic low back pain may find, some months after starting the drug, that he now craves the drug, wants more than is prescribed, operates a motor vehicle despite the effects of the drug, and is frequently borrowing opioids from friends and family or obtaining them through illicit means. Most clinicians would consider this reflective of opioid addiction, although at least some pain physicians suggest that acts such as prescription forgery and theft of drugs from others may represent consequences of undertreatment of pain [15].

Dependence Implies that a drug's presence is required for normal function and that abrupt dose reduction or cessation will result in withdrawal symptoms, depending on the class of drugs involved. While physical dependence is generally implied, the term psychological dependence is often used and is generally meant to convey craving for a drug, either for its pleasurable effects or to avoid the prospect of withdrawal symptoms. An individual prescribed highdose opioids for disseminated bony metastases would likely exhibit physiological and possibly even psychological dependence but would not be said to be suffering from addiction.

Opioid Withdrawal Is an extremely unpleasant condition lasting several days and sometimes likened to severe influenza, with prominent features including abdominal pain, nausea, diarrhea, mydriasis, lacrimation, and generalized piloerection [16]. The emergence of these symptoms following abrupt reductions in the dose of opioids, and their resolution following readministration of the drug, makes it difficult for even highly motivated patients who are dependent on opioids to reduce or eliminate opioid use without careful medical oversight and support.

Tolerance Is a reversible physiologic response to the persistent presence of agonists at various receptors within the central nervous system, most often resulting from the regular use of opioids, benzodiazepines, or alcohol. The term denotes adaptation to a drug's effects leading to a diminution of effects despite a constant dose or concentration [17]. Opioid tolerance is an expected consequence of long-term therapy, particularly at higher dose, although the mechanisms by which it occurs remain incompletely understood [18]. For example, the analgesia experienced by an individual following the initiation of opioids for chronic neuropathic pain is often greater than that experienced some months later if the dose is unchanged.

Pseudoaddiction The term pseudoaddiction was introduced in 1989 by Weissman and Haddox to reflect an "iatrogenic syndrome of abnormal behavior developing as a direct consequence of inadequate pain management" [19]. The concept holds that inadequate analgesia can lead a patient to demand higher doses of opioids, often employing behavioral changes to convince prescribers of the pain's legitimacy, and that this results in the erosion of trust between the patient and caregivers. For example, a patient with pain due to bony metastases who presents to physicians who refuse to prescribe opioids or who prescribe them at low doses may seek care from multiple physicians or ask for higher doses. These behaviors can be misinterpreted as signs of addiction, when in fact the patient is simply seeking adequate analgesia. It has been suggested that pseudoaddiction can be differentiated from addiction in part because the pseudoaddiction resolves with dose increases, whereas addiction remains the same or worsens [20].

Although these terms are often perceived as synonymous with hazardous use of opioids, it bears mention that patients can suffer serious opioid-related harm, including death, 
despite never purposefully misusing an opioid. For example, reports describe opioid-naïve patients prescribed an inappropriately high dose resulting in a fatal overdose after the first use of the drug [21]. More commonly, some patients with chronic pain may have their dose escalated too rapidly by a well-meaning physician and succumb to respiratory depression in the setting of a concomitant illness such as an chronic obstructive pulmonary disease or pneumonia [22, 23]. However, such instances are relatively uncommon, and most serious opioid-related harm accrues to patients who abuse opioids or become addicted to them.

\section{Evidence Regarding the Risk of Opioid Addiction}

Randomized trials of opioid therapy typically last only 4 to 8 few weeks, although a few last 16 weeks and one extended to 32 weeks [24]. These trials are designed to explore efficacy rather than safety, tend to exclude patients at high risk of opioid abuse, and employ doses lower than commonly seen in clinical practice. As a result, they offer little insight into the adverse consequences of chronic opioid therapy in routine clinical practice, and most of what is known regarding the addictive potential of chronic opioid therapy drives from case series, cross-sectional studies, and a small number of retrospective cohort studies.

\section{Studies Suggesting a Relatively Low Risk of Addiction}

In a cross-sectional study conducted in the mid-1970s, Medina and Diamond studied 2,369 patients with chronic headaches and identified 62 taking potentially addictive medications (opioids or barbiturate-analgesic combinations) for 4 or more days per week for at least 6 months [25]. Patients were classified as "abusers" if no symptoms of psychological or physical dependence were apparent, but the dose exceeded by at least $50 \%$ the maximal recommended dose in the 1975 edition of the Physicians' Desk Reference. In the sample, only 23 patients took opioids. One of six patients taking propoxyphene was deemed an "abuser," as were 2 of 15 patients taking codeine. While the definition of abuse employed by the investigators is easily criticized, even a superficial assessment of this study reveals that it provides no legitimate assessment of the actual risk of addiction during chronic opioid therapy. Nevertheless, the study has been repeatedly portrayed by others as providing empirical evidence of a low rate of opioid abuse, based on the observation that only three cases of abuse were identified in a sample of 2,369 patients [26-28].

In 1980, Porter and Jick [29] published a brief correspondence in The New England Journal of Medicine in which they described an examination of the medical records of 39,946 hospitalized medical patients. In this sample, 11,882 (28\%) received at least one prescription for an opioid, and among those with no previous history of addiction, the investigators identified addiction in just four patients, with "major" addiction in only one. Interpretation of this report is highly problematic, in part because the methods are not described in any detail. The report consists of just five sentences, and the definition of addiction (and, by extension, a previous history of addiction) used by the investigators was not reported. Although their methods are unclear, the report suggests that the investigators did not directly interview patients but instead based their conclusions on a retrospective review of patient files. Moreover, the conclusions were drawn from medical and psychiatric inpatients at only six hospitals [30] during an era in which chronic opioid therapy for noncancer pain was not commonplace. The generalizability to outpatients three decades later is unknown, but the brief correspondence has nevertheless been cited hundreds of times.

Another heavily cited study supporting the effective and safe use of opioids for chronic noncancer pain was published in 1986. In what many consider the seminal article on the topic, Portenoy and Foley [31] described a retrospective study of 38 patients who received care at a specialized pain clinic, most often for chronic back pain $(n=14)$ or chronic facial, abdominal, extremity or pelvic pain $(n=9)$. Of the patients in the series, $19(50 \%)$ had been treated with opioids for 4 or more years while six (16\%) had been treated for 7 years. Oxycodone, methadone, and levorphanol were the most commonly prescribed opioids. The majority of patients experienced adequate or partial analgesia, and only two patients (both with a history of substance abuse) displayed aberrant behaviors.

An important and often overlooked aspect of this case series is that two-thirds of subjects were maintained on less than $20 \mathrm{mg}$ of intramuscular morphine (or equivalent) per day, equivalent to approximately 40 to $60 \mathrm{mg}$ of oral morphine. This contrasts starkly with the doses commonly employed today. In one recent study of chronic opioid recipients in Ontario, Canada, $32.8 \%$ were prescribed more than $200 \mathrm{mg}$ or morphine, or equivalent, daily [32]. While the study by Portenoy and Foley demonstrates that low- to moderate-dose opioids can be an effective and safe option for carefully selected patients with chronic noncancer pain in a specialized setting, it is not possible to extrapolate their conclusions regarding aberrant behavior to patients receiving far higher doses in routine clinical practice.

In 1988, Tennant and colleagues reported on a pilot program involving 52 patients, the majority of whom had severe, intractable pain from arthritis or following trauma or surgery [33]. Varying doses of opioids (chiefly codeine or methadone) were used in conjunction with adjuvant treatments, resulting in adequate pain control for the majority of patients. Although the authors indicate that dependence developed in all patients, rates of addiction were not 
reported. Regardless, the study has been cited as providing evidence that chronic opioid therapy can be beneficial with little or no risk of addiction [34].

The uncritical and selective interpretation of these studies helped entrench the notion that addiction during chronic opioid therapy was rare. As noted earlier, this view was aggressively communicated to physicians by pharmaceutical manufacturers [4] and solidified by the imprimatur of national organizations such as the AAPM and APS, who jointly issued a four-page consensus statement that included the simple, unreferenced statement that the risk of addiction during chronic opioid therapy was low [35]. Many clinicians continue to hold this belief today.

\section{Studies Suggesting a Relatively High Risk of Addiction}

Primary care physicians need to be able to estimate the risk of opioid addiction in relatively unselected patients. Ideally, physicians who prescribe chronic opioid therapy would also have tools they could use to individualize risk estimates. Such tools, if properly validated, would allow physicians to differentiate between patients who can be safely prescribed long-term opioid therapy and those in whom the risks of chronic opioid therapy are likely to outweigh benefits. Understanding the risk factors and prevalence of addiction is also a prerequisite to proper counseling and obtaining informed consent prior to treatment. Even though it is obvious to those who understand the pharmacology of opioids, it is worth noting that physiologic dependence arises in virtually all patients who are treated with chronic opioid therapy [33, 36].

The incidence and prevalence of abuse and addiction will obviously vary substantially from one setting to another and will depend not only on the characteristics of a particular patient population but also on the propensity of a physician to prescribe opioids and the doses used. Unfortunately, there are very few large-scale epidemiologic studies in primary care settings that can be used to reliably determine the risk of addiction. Perhaps the most generalizable evidence comes from Boscarino et al. in Pennsylvania, who randomly selected more than 2,000 patients being treated at one of the nine primary care clinics or three specialty care clinics and administered structured diagnostic interviews to patients who agreed to participate [37]. Survey results were weighted to account for differences in baseline characteristics between respondents and nonrespondents. The authors reported that $35 \%$ of longer term opioid users (defined as those who received four or more prescriptions in the last 12 months) met DSM-5 criteria for a current or previous opioid use disorder. Similar results were obtained when using the DSM-4 criteria. It is worth noting that in this study, the risk of addiction was significantly higher in patients who reported higher than average pain levels and pain impairment but did not vary according to clinic setting, education, income, marital status, or gender. A limitation of this study is that it can sometimes be difficult to distinguish addiction from pseudoaddiction; the extent to which the investigators were able to do so is unclear.

In 2007, Martell and colleagues published a systematic review of the use of opioid for chronic back pain. In four short-term randomized controlled trials, analgesia with opioids was comparable to either active treatment or placebo. In the few (mostly cross-sectional) studies that reported the prevalence of aberrant medication-taking behaviors, estimates ranged from 5 to $24 \%$. However, only one of the seven studies addressing this issue had an acceptable quality score, and only two used a comprehensive, structured assessment of behaviors related to opioid dependence.

In 2008, Fishbain and colleagues performed a structured evidence-based review of 67 studies of opioid use for noncancer pain meeting prespecified quality metrics [38]. Among patients with at least 1 month of opioid therapy, they found an overall addiction rate of $3.3 \%$, while among patients with no previous opioid exposure, the rate was $4.4 \%$. Aberrant drug-related behaviors were detected in $11.5 \%$. However, Chou and colleagues [39] examined the evidence regarding aberrant drug-related behaviors during chronic opioid therapy. They noted that definitions of aberrant drug-related behaviors were not consistent across studies and did not account for the seriousness of identified behaviors. For example, excessive phone calls regarding opioids, lost prescriptions, "doctor shopping," detection of nonprescribed opioids on urine toxicology screening and criminal activity involving opioids were variously considered aberrant drug-seeking behaviors.

\section{Risk Factors for Opioid Addiction in Clinical Practice}

Predicting which patients will develop addiction to opioids is difficult. One screening tool that is reasonably widely used is the Opioid Risk Tool [40], a ten-item scale developed to help physicians determine which patients are high risk, moderate risk, and low risk of developing aberrant behaviors when prescribed chronic opioid therapy. Unfortunately, the tool was not developed in a manner consistent with recommended methodological standards [41]. For example, it was not validated in a separate population, and even within the derivation sample, $28 \%$ of those deemed to be at moderate risk developed one or more aberrant behaviors after 12 months. Many physicians would view this risk as unacceptably high, especially given the limited evidence of efficacy for chronic opioid therapy. In addition, the tool has limited face validity. For example, a 30-year-old woman with a history of alcohol abuse and depression would be classified as low risk. 
Because only 18 patients in the study were classified as low risk, a much larger study, ideally in a primary care setting, would be required to determine whether a patient deemed to be low risk according to the Opioid Risk Tool is in fact truly at low risk. To our knowledge, the Opioid Risk Tool has not been undergone formal impact analysis. Without such an assessment, physicians have no way of knowing whether using the tool is beneficial or harmful [42]. Despite these limitations, the Opioid Risk Tool has been prematurely incorporated into clinical practice guidelines [43] and point of care tools including the Opioid Manager (available at $\mathrm{http} / / /$ nationalpaincentre.memaster.ca/opioidmanager).

The Opioid Risk Tool does illustrate that prediction of opioid abuse is a highly inexact science. Much research remains to be done in this area. The available evidence suggests that risk factors for opioid abuse include a feeling of euphoria during use [44], a diagnosis of other substance abuse, mental health disorders, male sex, and younger age [45]. A thorough exposition of this issue and strategies to prevent opioid abuse have recently been published by Sehgal et al. [46].

\section{Conclusion}

A major reason why opioids have been prescribed increasingly frequently for chronic noncancer pain in North America over the past 25 years is the belief that addiction is a rare consequence of long-term opioid therapy. However, the limited data supporting that belief are of poor quality and not applicable to present-day patterns of opioid use. More recent and more rigorous studies suggest that opioid use disorders occur in up to one-third of patients on chronic opioid therapy.

The treatment of patients with chronic pain is a vexing problem, and medications are only one element of what should usually be a multimodal care plan. Prescribing opioids to maximize benefit while minimizing harm requires an accurate appreciation for the risks of addiction and its consequences. While additional research in this area is needed, clinicians should discard the notion that addiction is a rare consequence of long-term opioid therapy.

Acknowledgements We are indebted to Ashif Kachra for assistance with manuscript preparation.

\section{References}

1. Von Korff M, Kolodny A, Deyo RA, Chou R (2011) Long-term opioid therapy reconsidered. Ann Intern Med 155:325-328

2. Passik SD (2009) Issues in long-term opioid therapy: unmet needs, risks, and solutions. Mayo Clin Proc 84:593-601
3. Ballantyne JC (2007) Opioid analgesia: perspectives on right use and utility. Pain Physician 10:479-491

4. Dhalla IA, Persaud N, Juurlink DN (2011) Facing up to the prescription opioid crisis. BMJ 343:d5142

5. Ballantyne JC (2007) Opioid analgesia: perspectives on right use and utility. Pain Physician 10:479-491

6. Anonymous (1997) The use of opioids for the treatment of chronic pain. A consensus statement from the American Academy of Pain Medicine and the American Pain Society. Clin J Pain 13:6-8

7. Ballantyne JC (2007) Opioid analgesia: perspectives on right use and utility. Pain Physician 10:479-491

8. Lanser P, Gesell S (2001) Pain management: the fifth vital sign. Health Benchmarks 8(68-70):62

9. Booth M (1999) Opium: a history. St Martin, New York

10. Towns CB (1915) Habits that handicap. The menace of opium, alcohol and tobacco and the remedy, 1st edn. The Century Company, New York

11. Fishbain DA, Rosomoff HL, Rosomoff RS (1992) Drug abuse, dependence, and addiction in chronic pain patients. Clin J Pain 8:77-85

12. Hernandez SH, Nelson LS (2010) Prescription drug abuse: insight into the epidemic. Clin Pharmacol Ther 88:307-317

13. Center for Substance Abuse Treatment (2006) Prescription medications: misuse, abuse, dependence and addiction. Substance Abuse Treatment Advisory 5:1-4

14. Compton P, Darakjian J, Miotto K (1998) Screening for addiction in patients with chronic pain and "problematic" substance use: evaluation of a pilot assessment tool. J Pain Symptom Manage $16: 355-363$

15. Fisher FB (2004) Interpretation of aberrant drug-related behaviors. J Am Phys Surg 9:25-28

16. Kosten TR, O'Connor PG (2003) Management of drug and alcohol withdrawal. N Engl J Med 348:1786-1795

17. Sehgal N, Manchikanti L, Smith HS (2012) Prescription opioid abuse in chronic pain: a review of opioid abuse predictors and strategies to curb opioid abuse. Pain Physician 15: ES67-ES92

18. Koch T, Hollt V (2008) Role of receptor internalization in opioid tolerance and dependence. Pharmacol Ther 117:199-206

19. Weissman DE, Haddox JD (1989) Opioid pseudoaddiction - an iatrogenic syndrome. Pain 36:363-366

20. Kahan M, Srivastava A, Wilson L, Gourlay D, Midmer D (2006) Misuse of and dependence on opioids: study of chronic pain patients. Can Fam Physician 52:1081-1087

21. Jumbelic MI (2010) Deaths with transdermal fentanyl patches. Am J Forensic Med Pathol 31:18-21

22. Darke S, Duflou J, Torok M (2011) Toxicology and characteristics of fatal oxycodone toxicity cases in New South Wales, Australia 1999-2008. J Forensic Sci 56:690-693

23. Darke S, Kaye S, Duflou J (2006) Systemic disease among cases of fatal opioid toxicity. Addiction 101:1299-1305

24. Ballantyne JC (2007) Opioid analgesia: perspectives on right use and utility. Pain Physician 10:479-491

25. Medina JL, Diamond S (1977) Drug dependency in patients with chronic headaches. Headache 17:12-14

26. Lipman AG, Jackson KC II (2004) Opioid pharmacotherapy. In: Warfield CA, Bajwa ZA (eds) Principles and practice of pain medicine, 2nd edn. McGraw-Hill, New York, pp 583-600

27. Portenoy RK, Foley KM (1986) Chronic use of opioid analgesics in non-malignant pain: report of 38 cases. Pain 25:171-186

28. Adams NJ, Plane MB, Fleming MF, Mundt MP, Saunders LA, Stauffacher EA (2001) Opioids and the treatment of chronic pain in a primary care sample. J Pain Symptom Manage 22:791-796

29. Porter J, Jick H (1980) Addiction rare in patients treated with narcotics. N Engl J Med 302:123

30. Jick H (1970) COmprehensive drug surveillance. JAMA 213:1455-1460 
31. Portenoy RK, Foley KM (1986) Chronic use of opioid analgesics in non-malignant pain: report of 38 cases. Pain 25:171-186

32. Gomes T, Juurlink DN, Dhalla IA, Mailis-Gagnon A, Paterson JM, Mamdani MM (2011) Trends in opioid use and dosing among socio-economically disadvantaged patients. Open Med 5:e13-e22

33. Tennant F Jr, Robinson D, Sagherian A, Seecof R (1988) Chronic opioid treatment of intractable, non-malignant pain. NIDA Res Monogr 81:174-180

34. Fishbain DA, Cole B, Lewis J, Rosomoff HL, Rosomoff RS (2008) What percentage of chronic nonmalignant pain patients exposed to chronic opioid analgesic therapy develop abuse/addiction and/or aberrant drug-related behaviors? A structured evidence-based review. Pain Med 9:444-459

35. Anonymous (1997) The use of opioids for the treatment of chronic pain. A consensus statement from the American Academy of Pain Medicine and the American Pain Society. Clin J Pain 13: 6-8

36. Ballantyne JC (2007) Opioid analgesia: perspectives on right use and utility. Pain Physician 10:479-491

37. Boscarino JA, Rukstalis MR, Hoffman SN et al (2011) Prevalence of prescription opioid-use disorder among chronic pain patients: comparison of the DSM-5 vs. DSM-4 diagnostic criteria. J Addict Dis 30:185-194

38. Fishbain DA, Cole B, Lewis J, Rosomoff HL, Rosomoff RS (2008) What percentage of chronic nonmalignant pain patients exposed to chronic opioid analgesic therapy develop abuse/addiction and/or aberrant drug-related behaviors? A structured evidence-based review. Pain Med 9:444-459
39. Chou R, Fanciullo GJ, Fine PG, Miaskowski C, Passik SD, Portenoy RK (2009) Opioids for chronic noncancer pain: prediction and identification of aberrant drug-related behaviors: a review of the evidence for an American Pain Society and American Academy of Pain Medicine clinical practice guideline. J Pain 10:131-146

40. Webster LR, Webster RM (2005) Predicting aberrant behaviors in opioid-treated patients: preliminary validation of the Opioid Risk Tool. Pain Med 6:432-442

41. McGinn TG, Guyatt GH, Wyer PC et al (2000) Users' guides to the medical literature: Xxii: how to use articles about clinical decision rules. JAMA 284:79-84

42. Reilly BM, Evans AT (2006) Translating clinical research into clinical practice: impact of using prediction rules to make decisions. Ann Intern Med 144:201-209

43. Furlan AD, Reardon R, Weppler C (2010) Opioids for chronic noncancer pain: a new Canadian practice guideline. CMAJ 182:923-930

44. Bieber CM, Fernandez K, Borsook D et al (2008) Retrospective accounts of initial subjective effects of opioids in patients treated for pain who do or do not develop opioid addiction: a pilot casecontrol study. Exp Clin Psychopharmacol 16:429-434

45. Edlund MJ, Steffick D, Hudson T, Harris KM, Sullivan M (2007) Risk factors for clinically recognized opioid abuse and dependence among veterans using opioids for chronic non-cancer pain. Pain 129:355-362

46. Sehgal N, Manchikanti L, Smith HS (2012) Prescription opioid abuse in chronic pain: a review of opioid abuse predictors and strategies to curb opioid abuse. Pain Physician 15: ES67-ES92 